\title{
La IAP en las ciencias sociales y su diversidad en la producción científica situada
}

\section{The PAR in the social sciences and its diversity in the scientific production situated}

\author{
Néstor García Montes \\ Universidad Complutense de Madrid, España \\ nestorga@ucm.es \\ María José Díaz Santiago \\ Universidad Complutense de Madrid, España \\ mjdiaz@cps.ucm.es
}

\begin{abstract}
Resumen: El artículo reflexiona sobre la importancia de las metodologías IAP dentro de las ciencias sociales. Ubicándola más allá de las metodologías tradicionales, éste muestra la singularidad de esta metodología desde su génesis, que aparece con el objetivo de la participación ciudadana en el proceso de investigación, a través de la aplicación de una pluralidad de técnicas y herramientas que, usadas de forma combinada, favorecen debates y transformaciones sociales situadas del sujeto en proceso.

A partir de su desarrollo histórico y territorial, así como de su variedad científica, el artículo realiza un análisis ontológico y deontológico que termina con una breve explicación de una selección de ocho artículos que reflexionan sobre las posibilidades de esta metodología.
\end{abstract}

Palabras clave: Investigación-acción-participativa, metodología, ciencias sociales

Abstract: Article reflects on the importance of PAR (IAP) methodologies within the social sciences. Locating it beyond traditional methodologies, this shows the uniqueness of this methodology from its genesis, which appears with the aim of citizen participation in the research process, through of the application of a plurality of techniques and tools that, used in combination, favor debates and situated social transformations of the subject in process.

Based on its historical and territorial development, as well as its scientific variety, the article carries out an ontological and deontological analysis that ends with a brief explanation of a selection of eight articles that reflect on the possibilities of this methodology.

Keywords: Participatory Action Research, methodology, social sciences 


\section{INTRODUCCIÓN}

Podemos encontrar el principal antecedente a la Investigación-acción participativa en la propuesta de Lewin sobre la action-research (Lewin, 1946), que la entendía como un modelo investigativo basado en el cuestionamiento autorreflexivo desarrollado por los propios sujetos implicados para la mejora de sus circunstancias. Se trata, por tanto, de promover la intervención y transformación dentro de los grupos sociales, organizaciones o comunidades desde la participación directa de las mismas. Lewin realizó estudios en contextos organizacionales, laborales, educativos y comunitarios, muchos de ellos en el marco del Instituto Taviskov, desde la lógica del autoaprendizaje, la autorreflexión y la autotransformación a partir de las prácticas de las propias personas interesadas. Sin embargo, se puede encontrar algún precedente histórico a la implicación participativa en el análisis de su realidad por parte de los sujetos afectados en la obra de John Howard o de Charles Booth. El primero fue un reformador del sistema carcelario británico en el s. XVIII, considerado por algunos como el alma mater del derecho penitenciario (Gudín, 2005). Howard (1726-1790) trató de humanizar las prisiones con la intervención de los propios reclusos para que su estancia fuera más digna. Un siglo después, Booth (1840-1916), también británico, realizó importantes estudios sobre la pobreza en Londres (Booth, 2012 [1889]), ampliando la información cuantitativa y estadística con métodos cualitativos para captar la realidad social desde la experiencia y vivencia de los sujetos (Gallino, 1995), y sumergiendo a un equipo de investigación en labores etnográficas de contacto directo con la población objeto de estudio.

La denominación Investigación-Acción Participativa (IAP) se empezó a extender desde finales de la década de 1960, especialmente en América Latina, de la mano de las luchas populares que acontecían y la educación popular de Freire (1967). Fals Borda también contribuyó notablemente a la práctica participativa en la investigación social con campesinos, obreros e indígenas, y fue el máximo responsable de uno de los principales hitos de la IAP, el I Congreso Mundial de Investigación-Acción y Análisis Científico celebrado en 1977 en su país natal, en Cartagena de Indias (Colombia). En las dos ediciones posteriores que se han celebrado de este Simposio, veinte y cuarenta años después de la primera, participó Tomás R. Villasante, una de las figuras españolas más reconocidas en las metodologías participativas. En 1995 publicó Las democracias participativas y hasta la fecha sigue realizando aportaciones innovadoras a las ciencias sociales desde su propuesta de Socio-praxis (Villasante, 2006), lo que le ha convertido en un referente nacional e internacional.

La IAP se puede definir como «un método de estudio y acción que busca obtener resultados fiables y útiles para mejorar situaciones colectivas, basando la investigación 
en la participación de los propios colectivos a investigar» (Alberich, 2009: 10). El investigador se convierte, junto con las personas investigadas, en agentes de cambio desde la reflexión (investigación) y la acción (praxis). Esta perspectiva confronta críticamente al positivismo, ya que requiere del concurso del objeto de investigación, quebrando la separación clásica entre objeto y sujeto de estudio para derivar en una relación sujeto-sujeto, entendido éste como sujeto-en proceso (Colectivo IOÉ, 2003). La población analizada se erige en protagonista de la construcción del conocimiento de la realidad social que les atañe, en función de cómo dicha realidad interpela a los individuos, desde su subjetividad, pero a través de la acción de objetivarla. En palabras de Montañés: «Toda realidad surge de la actividad objetivadora del sujeto. Y ésta nace de la capacidad antropológica reflexiva de representarnos representándonos la realidad que nos representamos. Dicho de manera coloquial, de la capacidad de vernos viendo la realidad que vemos» (Montañés, 2008: 174). Los sujetos de investigación y el propio investigador, como seres en situación (Freire, 1983), se comprenden y actúan desde la percepción in situ del contexto y la situación existencial que experimentan. Para Lewin, la percepción social (la autointerpretación de la realidad) es lo que conduce a la acción desde procesos grupales (Lewin, 1948). La búsqueda de la visión «subjetiva» de los fenómenos sociales por parte de la IAP, frente a la visión exclusivamente «objetivista», técnica o científica, y la concepción del ser humano como producto y productor de la realidad social (Bourdieu, 1997) pueden trazar puentes con las representaciones colectivas de Durkheim, la psicología cognitiva (Broadbent, Neisser y Bruner), las representaciones sociales (Moscovici), la fenomenología (Husserl y Schutz), la construcción social de la realidad (Berger y Luckman), la geografía de la percepción y del comportamiento (Lynch) o la etnometodología (Garfinkel).

En términos epistemológicos, la IAP propicia la simetría entre la persona que investiga y la persona investigada. La primera ya no es un observador separado (y en un plano de superioridad) de aquello que observa, y la segunda ya no es un elemento de análisis ex cathedra en un «laboratorio» y adquiere entidad proactiva y consciente para producir conocimiento (para el investigador y para sí mismo). En este sentido, se marca una considerable diferencia con la investigación desde enfoques cuantitativos y cualitativos clásicos, en los que suele ser habitual la apropiación y el monopolio del proceso investigativo y de los resultados por el experto o científico (De Schutter, 1983). No se investiga «a» un determinado grupo social, sino que se investiga «con» (Whyte, 1991) y «para» ese grupo social. Como señalaba Fals Borda (1979), investigador e investigado, anulando la separación entre ambos, persiguen un objetivo que ha sido definido conjuntamente desde la autoconstrucción de las preguntas de investigación, partiendo 
del análisis histórico de la comunidad local o grupos afectados desde la percepción subjetiva.

Por tanto, la IAP abre una línea de investigación que prioriza la praxis (y se teoriza sobre la práctica), la transformación social desde la reflexión autocrítica y la acción de los propios sujetos implicados frente al conocimiento pasivo, y la conexión con la realidad y la situación conflictiva de los sectores más desfavorecidos (en sintonía con la pedagogía del oprimido de Freire), imbuyendo al científico en dicha realidad frente a la observación distante y desafectiva del positivismo social. Además, ofrece la articulación de un conjunto de estrategias y métodos que permitan a los sectores populares analizar, planificar y actuar sobre sus condiciones de vida (Chambers, 1994). Una metodología y unas herramientas de investigación que gravitan en una lógica procesual y espiral de observación-análisis-autodiagnóstico-reflexión-devolución-planificación-acción-evaluación. Y aunque han coexistido diferentes escuelas y enfoques de la investigación-acción, con diversas denominaciones y etiquetas, muchas veces ignorantes unas de otras o con disparidades sociocontextuales (Brown y Tandon, 1978), en el frontispicio ético se encuentra la democracia participativa como paradigma de un modelo de ciudadanía activa, crítica, emancipada y consciente.

\section{LA PRODUCCIÓN CIENTÍFICA SOBRE IAP Y LA NECESIDAD DE HACER UN MONOGRÁFICO}

Esa gran diversidad en la investigación y la necesidad del incluir a ese sujeto en proceso en la misma es lo que ha hecho que nos planteemos la necesidad de hacer un monográfico, que dé cuenta de esa gran producción científica de la IAP en cuanto a la temática, pero, también, en relación a su poder de transformación social para los grupos, comunidades o sociedades a las que se le ha aplicado.

En la última década se ha producido un creciente interés por la participación e implicación de los sujetos tanto en los procesos de gestión y políticas públicas, impulsados desde las Administraciones y las instituciones de gobierno, especialmente local (García-Montes, 2019), como en los procesos de investigación desde esferas académicas. La IAP ha encontrado un campo de aplicación empírica, desde una lógica de transformación social, en la acción local, entendida ésta tanto en términos territoriales como en términos de iniciativas y proyectos de ámbito cotidiano y acotado. Estamos comprobando cómo, especialmente desde la crisis de legitimidad de los partidos políticos tradicionales (Hernández, 2012), la democracia participativa, alentada por metodologías como 
la IAP, ocupan un espacio de atención mayor en la actuación pública de arriba abajo y en la actuación de base social de abajo arriba.

En cuanto a la primera, la actuación institucional favoreciendo políticas públicas con espacios participativos, convendría aclarar que tales procesos no siempre son todo lo participativos y transformadores que deberían. No cabe obviar que en muchas ocasiones se trata de estrategias políticas con fines de imagen más que con el objetivo de propiciar verdaderas prácticas de democracia participativa. Sin embargo, a pesar de la mayor o menor (o nula, en algunos casos) calidad, rigor, profundidad y compromiso real de dichos procesos, lo cierto es que se ha producido una tendencia a integrar la visión y opinión de la ciudadanía en la gestión pública (García-Montes, 2019), o en una parte de dicha gestión. En cuanto a la actuación de base social, de abajo arriba, son crecientes las propuestas y experiencias llevadas a cabo por movimientos sociales, colectivos informales, cooperativas, asociaciones, grupos autoorganizados y comunidades vecinales para, al margen de la tutela, impulso o patrocinio de las Administraciones (experiencias en el ámbito educativo, agroecológico, convivencial, de las migraciones, de la equidad de género, de la identidad...). Son proyectos de participación social en los que también observamos la aplicación de metodologías participativas, como la IAP, con mayor o menor conocimiento, recursos y capacidad técnica, pero que plasman la presencia al alza de esta forma de investigar, poniendo en el centro al sujeto implicado como motor de conocimiento y de cambio, no sólo en la esfera académica o de las políticas públicas institucionales, sino también en la calle, en los barrios, en los colectivos, en las asociaciones, en definitiva, en la sociedad civil.

Un ejemplo de lo aquí expuesto es este monográfico, donde se recoge una panoplia de investigaciones actuales, que rompen con las metodologías tradicionales cuantitativa y cualitativa, donde el objeto investigado no participa de la misma, por otra, que lo incorpora, es decir, lo convierte en sujeto en proceso de conocimiento.

De la IAP se puede decir que es variada, rica, local y participada. Sin embargo, todavía es escasa su producción académica, a pesar de su capacidad de integración y de innovación social en un momento donde las crisis se muestran cada vez más recurrentes, impredecibles e invisibles, donde las transformaciones sociales se producen en línea con un mundo lleno de contradicciones que en lo cotidiano pueden generar actos, de por sí, de desbordes (Villasante, 2014).

Su pluralidad en el uso de técnicas para captar cualquier tipo de información y planificar el escenario de investigación, a través de procesos compartidos donde la devolución al destinatario genera que este lo haga suyo, integrando una multiplicidad de know-hows en lo cotidiano. A través de la reflexividad muestra cómo las relaciones sujeto-sujeto en la investigación e interacción social son fuente de creatividad ante las resistencias, indi- 
viduales o colectivas, conscientes o inconscientes, que parten de un conocimiento situado (Haraway, 1995) donde prima la dimensión de conflicto oprimido-opresor.

La capacidad de agencia (Ibáñez, 1986), motivada por la comprensión de la realidad, da cuenta de los valores y las emociones de los sujetos en procesos, que nos hace interrogar no sólo sobre el porqué de la IAP, sino por su hacer. Por sus límites políticos, económicos y sociales, pero también por su capacidad de activismo y de investigación en profundizar cómo la IAP reconoce las estructuras económicas y políticas, así como sus estructuras de género.

De ahí que hayamos considerado necesario este monográfico, en un momento donde la pluralidad y la especificidad de grupos muestran relaciones heterogéneas vivenciales, de opresión, resistencia y empoderamiento, o, mejor dicho, de superación. La pluralidad de técnicas y herramientas adaptadas a esa diversidad de temas muestra la capacidad de transformación social en un momento donde el sufrimiento de las personas es mayor y es necesario colectivizar sus miedos y liberaciones.

\section{SOBRE EL MONOGRÁFICO: OBJETIVOS Y ESTRUCTURA}

Un monográfico sobre IAP, tautológicamente, como indica el acrónimo, intenta mostrar la investigación, acción y participación que, como metodología, se viene desarrollando y que, como en este artículo se aboga, refleja la preocupación de parte de la ciudadanía por las transformaciones sociales que se están desplegando en un periodo de aceleración cíclica de crisis. La capacidad de mostrar los tropos que guarda el sistema a fin de mantener las relaciones asimétricas de poder y que ésta muestra a través de metáforas, metonimias, sinécdoques u otros recursos discursivos hace de esta metodología un método apropiado para entrever las sombras que se generan dentro del sistema. Además, su capacidad de visibilizar ideología o símbolos que construyen la interacción con los demás y/o con uno mismo y que aparecen invisibilizadas por la naturalización y sustantivadas por las acciones de grupos diversos o la psicologización imperante que incapacitan la agencia es elaborada a través de la reflexividad colectiva que produce esta metodología a partir de una pluralidad de técnicas y herramientas que, utilizadas de forma individualizada o conjunta, muestran la riqueza que aporta dicha metodología.

En ese sentido, el objetivo que nos hemos marcado con este monográfico es dar a conocer algunas de las investigaciones que se están realizando en torno a la IAP, con el pretexto de reflexionar críticamente sobre sus posibilidades metodológicas y, también, políticas. A partir de este objetivo se conocerá parte de la pluralidad de técnicas y herramientas que se utilizan en la IAP y se muestra la gran diversidad temática que, sin em- 
bargo, concentra parte de las preocupaciones y capacidad de empoderamiento que muestra la ciudadanía.

Por ello, la estructura del monográfico responde a un acto meditado y reflexivo que lleva al lector y a la lectora a conocer la IAP, a través de sus múltiples interrogantes, variedades temáticas y herramientas. La reflexividad que la caracteriza posibilita ahondar en temáticas tan complejas como la militancia, el aprendizaje, el feminismo, los posicionamientos políticos y la innovación social. En relación con esa estructura, nos gustaría señalar que, también, responde a un acto de devolución a la generosidad mostrada por uno de sus fundadores, Tomas R. Villasante, cuyo texto, realizado junto a Loli Hernández, hemos querido dejar para el final como colofón de los aprendizajes y desbordes (Villasante, 2014) mostrados a lo largo del monográfico y que son muestra del saber hacer transmitido a través de su escuela.

El monográfico está formado por nueve artículos que se dividen en dos líneas argumentales: la primera, formado por este artículo y otros tres más, se encuadra dentro de la parte más teórica del mismo, centrado en el conocimiento metafísico y ético del método. Este artículo, como se ha podido vislumbrar, no tiene como pretensión, sólo, la de presentar el monográfico, sino de reflexionar sobre la IAP dentro de las ciencias sociales, ubicándola entre las metodologías y la producción científica existentes. A partir de él aparecen un grupo de artículos, como el realizado por uno de los coordinadores de este monográfico, Néstor García Montes, que, encuadrados, también, en la parte más teórica de la praxis metodológica de la IAP, se centran en el ámbito de la ética y política metodológica. El artículo «La oportunidad perdida para la gobernanza participativa y la sostenibilidad en el ámbito local: la Agenda 21», de Néstor García Montes, nos retrotrae al programa de la ONU para denunciar cómo éste se ha convertido en una asignatura pendiente para la participación y sostenibilidad en el ámbito local a pesar de las grandes perspectivas creadas en torno a la Agenda. En el segundo artículo, «¿La innovación social como método de investigación participativo?», de José Hernández-Ascanio, el autor se pregunta por la articulación de la IAP con diferentes prácticas de investigación que puedan considerarla como un método de innovación social generado en la sociopraxis. El último artículo, que englobaría la temática ética y política, «Trabajo Social y metodologías participativas: retos y oportunidades», de Antonio Álvarez-Benavides, trata de la necesidad de reformular la disciplina, una forma de innovación social, a partir de la incorporación como disciplina universitaria y que para el autor se produce con la introducción deontológica de la intervención a partir de la socio-praxis.

A partir de aquí aparecen cinco estudios de caso que utilizan diferentes técnicas y herramientas basadas en la IAP, mostrando la gran versatilidad metodológica, de transformación social y empoderamiento de la misma. Dos casos referentes a proyectos de 
innovación docente en el aula y tres casos que muestran el trabajo y el conocimiento creado dentro de los movimientos sociales. El artículo «Precariedad laboral universitaria con perspectiva de género a través de la IAP. El teatro INTERSOC como herramienta participativa de análisis en el aula», de Stribor Kuric Kardelis, María José Díaz Santiago, Pablo López Calle e Irene Pastor Bustamante, recoge la experiencia del Aula Laboratorio de Teatro Social con estudiantes universitarios que, a través de herramientas propias del arte escénico, reflexionan sobre su precariedad laboral, en una especie de «artivismo» universitario que hace confluir aprendizaje y acción. «La Investigación-acción participativa en la metodología docente universitaria. Una experiencia de innovación desde la perspectiva de género», de Irene Martínez Martín, Teresa Rabazas Romero y Ainhoa Resa Ocio, es otro de los artículos que muestra la importancia de la IAP en las metodologías de innovación docente universitaria, en este caso presentando cómo a partir de ella se puede introducir y evaluar la aplicación de la perspectiva de género dentro del aula.

Finalmente, el tercer grupo de trabajos que entraría dentro del bloque de casos, recoge tres artículos centrados en los movimientos sociales. El primero, «Revisando la Investigación Militante desde un enfoque aplicado en el contexto de los movimientos sociales», de Javier Ortega Fernández, trata del conocimiento generado desde el activismo cuya capacidad de comprensión se muestra fuera de las estancias académicas y que son reflejo de la necesidad de integración de ese conocimiento para la comprensión de las diferentes realidades sociales. El penúltimo artículo, «Multilemas y lógica hexagonal como herramienta para la creatividad social: sobre un caso de urbanismo participativo», de Javier Caballero Ferrándiz, se centra en la importancia del lenguaje en el urbanismo participativo, la riqueza que en su devolución trae contradicciones y subversión tanto en lo dicho como en aquello que no se dice. Por último, el artículo de Loli Hernández y Tomás R. Villasante, «Dispositivos frente a la hegemonía patriarcal, en procesos democrático-participativos», se centra en aspectos metodológicos de la IAP para la estrategia de transformación, donde a través del análisis de nueve aportaciones feministas, plantea argumentos y herramientas para poder superar las políticas patriarcales mediante la acción-reflexión-acción.

\section{BIBLIOGRAFÍA}

Alberich, T. (2009). «La Investigación-Acción Participativa, método y práctica», en IV Congreso Internacional sobre Investigación-Acción Participativa, octubre 2009, Valladolid (España). 
Bourdieu, P. (1997), Razones prácticas, Barcelona, Anagrama.

Booth, C. (2012 [1889]). Life and Labour of the People, Vol. I, Charleston (Carolina del Sur): Nabu Press.

Chambers, R. (1994). «The origin and practice of participatory rural appraisal», World Development, 22: 7, pp. 953-969.

Colectivo IOÉ (2003), «Investigación Acción Participativa: propuesta para un ejercicio activo de la ciudadanía», ponencia presentada en el Encuentro de la Consejería de Juventud Córdoba, junio de 2003.

De Schutter, A. (1983). Investigación participativa: una opción metodológica para la educación de adultos. Michoacán (México): CREFAL.

Díaz, M.J. y Pastor, I (2018). «Del becariado al precariado. Análisis de la precariedad laboral del alumnado universitario a través de metodologías participativas», en Investigación y prácticas sociológicas: Escenarios para la transformación social. Madrid: Editorial UNED.

Fals Borda, O. (1979). El problema de cómo investigar la realidad para transformarla por la praxis. Bogotá: Ediciones Tercer Mundo.

Freire, P. (1967). La educación como práctica de la libertad, Río de Janeiro, Paz e Terra.

- (1983). Pedagogía del oprimido, México, Siglo XXI.

Gallino, L. (1995). Diccionario de Sociología, México DF: Siglo XXI.

García-Montes, N. (2019). «Abriendo caminos. Los procesos de participación ciudadana promovidos a nivel institucional en el ámbito local, como escuela de profundización democrática». Forum Revista Departamento de Ciencia Política, Universidad Nacional de Colombia, n. ${ }^{\circ} 15$, pp. 11-35.

Gudín, F. (2005). «Crónica de la vida de John Howard, alma mater del derecho penitenciario», en $A D P C P$, vol. LVIII, pp. 95-170.

Haraway, D. (1995). «Conocimientos situados: la cuestión científica en el feminismo y el privilegio de la perspectiva parcial», en Ciencia, ciborgs y mujeres. La reinvención de la naturaleza, Madrid: Cátedra, pp. 313-346

Hernández, A. (2006). «Modelos de democracia liberal representativa: limitaciones y promesas incumplidas». Co-herencia, n. ${ }^{\circ}$ 3: 4, pp. 37-75.

Ibáñez, Jesús. (1986). «Perspectivas de La Investigación Social: El Diseño En Las Tres Perspectivas». En El Análisis de La Realidad Social. Métodos y Técnicas de Investigación Social, eds. Manuel García Ferrando, Jesús Ibáñez, and Francisco Alvira. Madrid: Alianza, 57-98.

Las Brown, L.D y Tandon, R. (1978). «Interviews as catalysts in a community setting». En Journal of Applied Psychology, 63, pp. 197-205. 
Lewin, K. (1946). «Action research and minority problems», en Journal of Social Issues, 2:4, pp. 34-46.

- (1948). Resolving Social Conflicts. New York: Harper and Row Publishers.

Montañés, M. (2008). «La Matriz reflexiva: justificación y procedimiento», en Martínez, Z. y Blas A. (coords.), Poder político y participación, Construyendo ciudadanía 11, Bilbao, Servicio Central de Publicaciones del Gobierno Vasco.

Villasante, T.R. (1995). Las democracias participativas. De la participación ciudadana a las alternativas de sociedad, Madrid, HOAC.

- (2006). «La Sociopraxis: un acoplamiento de metodologías implicativas», en Canales, M. (coord.), Metodología de Investigación Social, Santiago de Chile, LOM.

- (2014). Redes de vida desbordantes. Fundamentos para el cambio desde la vida cotidiana. Madrid: Editorial Catarata.

Whyte, W.F. (1991). «Participatory Action Research: Through Practice to Science in Social Research», en Whyte, W.F. (ed.), Participatory Action Research. California: SAGE Publications. 